\title{
PHASE UNWRAPPING AND DENOISING FOR TIME-OF-FLIGHT IMAGING USING GENERALIZED APPROXIMATE MESSAGE PASSING
}

\author{
Jonathan Mei, Ahmed Kirmani, Andrea Colaço, and Vivek K Goyal \\ Massachusetts Institute of Technology \\ Research Laboratory of Electronics
}

\begin{abstract}
We present a new method for simultaneously denoising and unwrapping phase in multi-frequency homodyne time-of-flight ranging for the formation of accurate depth maps despite low SNR of raw measurements. This is achieved with a new generalized approximate message passing (GAMP) algorithm for minimum mean-squared error estimation of the phase. A detailed, physically-accurate acquisition model is central in achieving high accuracy, and the use of the GAMP methodology allows low computational complexity despite dense dependencies and the nonlinearity and non-Gaussianity of the acquisition model. Numerical simulations demonstrate that our integrated approach performs better than separate unwrapping followed by denoising. This performance translates to lowering the optical power consumption of time-of-flight cameras for a fixed acquisition quality.
\end{abstract}

Index Terms - Belief propagation, generalized approximation message passing, phase unwrapping, range imaging, time-of-flight cameras, 3D capture.

\section{INTRODUCTION}

Measuring distances using the time-of-flight (ToF) of light is a wellestablished method that is implemented in a variety of technologies. LIDAR systems sequentially make many range (longitudinal) measurements by ToF; raster scanning by a laser light source produces the transverse spatial resolution to form a range image or depth map [1,2]. Homodyne ToF cameras make these measurements simultaneously with a diffuse light source and reflected light focused on an array of sensors, enabling real-time imaging at video rates [3-5]. Other methods for obtaining transverse spatial resolution have also recently been introduced $[6,7]$.

ToF systems use periodic light sources and hence suffer from periodic ambiguities in distance measurements-a phase unwrapping problem. Phase unwrapping of two-dimensional signals is a longstanding problem that arises in a variety of fields, including synthetic aperture radar, magnetic resonance imaging, and adaptive optics, as well as the ToF imaging methods of interest here [8]. Several methods are reviewed in [9], including those based on norm minimization [10], branch cuts [11], and network flows [12,13]. A more recent technique attempts to solve nonconvex integer programming problems and denoise after unwrapping [14].

The use of multiple modulation frequencies can aid in unwrapping by increasing the unambiguous distance [15-17] (see Section 2.3), but it is not a panacea. The amount of data collected

This material is based upon work supported in part by the National Science Foundation under Grant No. 1101147, the HP Labs Innovation Research Program, and a Qualcomm Innovation Fellowship. is proportional to the number of modulation frequencies, so the acquisition speed must be increased to maintain a fixed frame rate. Furthermore, the pixel-wise unwrapping problem is not easily or accurately solved at low SNR. Other changes to hardware are also possible, with various costs and benefits [18]. Phase unwrapping remains a significant challenge because it is difficult to effectively exploit domain knowledge about the signals and the acquisition physics while keeping the computational complexity low.

This paper introduces an integrated method for addressing phase unwrapping in ToF imaging based on loopy belief propagation (BP). The method simultaneously applies probabilistic modeling and optimal estimation to accomplish both unwrapping and denoising in a unified framework. Our solution maintains low complexity through the use of generalized approximate message passing (GAMP) [19, $20]$ and, using the same hardware architecture as existing ToF systems, achieves better performance than separate processing. Our approach applies with one or more modulation frequencies; we demonstrate it using two modulation frequencies.

The closest precedent to our work outside the context of ToF imaging is the use of GAMP for phase retrieval [21]. The connection is rather superficial in that the measurement model (and hence the derived GAMP algorithm) is very different than the one considered here. An additional novelty of our work is the use of GAMP with a vector-valued measurement for each scalar mixed variable; this highlights the generality of GAMP.

The rest of the paper is organized as follows: Section 2 describes the operation and noise modeling of time-of-flight cameras and the phase unwrapping problem that arises from using two modulation frequencies. Section 3 presents the theory behind the denoising and explains the structure of the problem. Section 4 compares the performance of our method to existing methods for separately unwrapping and denoising depth images. We conclude in Section 5.

\section{OPERATION OF TIME-OF-FLIGHT CAMERAS}

\subsection{Homodyne Measurement of Phase}

Fig. 1 shows the operation of an amplitude-modulated cosine wave homodyne ToF camera. The $T$-periodic source signal $s(t)=1+$ $\cos (2 \pi f t)$ with modulation frequency $f=1 / T$, generated from $T$-periodic reference signal $p(t)$, illuminates the entire scene. The scene return signal at pixel $i$ can be modeled as

$$
r_{i}(t)=a_{i} \cos \left(2 \pi f\left(t-\tau_{i}\right)\right)+\left(a_{i}+b_{i}\right),
$$

where $a_{i}$ is the attenuated amplitude of the reflected sinusoid, $c$ is the speed of light, $\tau_{i}=2 z_{i} / c$ is the time delay due to light travel from the camera to the scene point at distance $z_{i}$ and back to the sensor, and $b_{i}$ is the constant contribution from ambient light. 


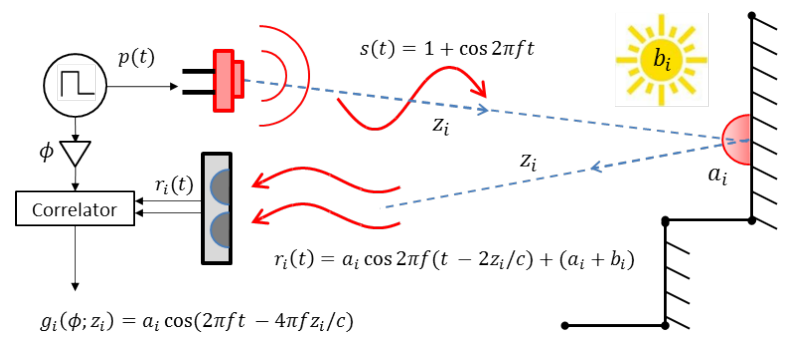

Fig. 1: Signal processing abstraction of homodyne ToF camera under differential mode operation.

The return signal $r_{i}(t)$ is correlated with the phase-shifted periodic reference signal $p(t+\phi)$ and a half-period shifted copy $p(t+$ $\phi+T / 2)$. The phase shift $\phi$ is of interest, as will be demonstrated shortly. The resulting correlation functions are subtracted (see [22] for details), and the final measured output is a function of $\phi$ given by

$$
g_{i}\left(\phi ; z_{i}\right)=a_{i} \cos \left(2 \pi f \phi-4 \pi f z_{i} / c\right) .
$$

Thus by varying the phase shift $\phi$ of the reference signal $p(t+\phi)$, we can effectively sample the correlation function $g_{i}\left(\phi ; z_{i}\right)$.

Estimating the amplitude of the sinusoid and the distance requires at least two samples in $\phi$ per period of $g_{i}\left(\phi ; z_{i}\right)$ at fixed frequency $f$. However, typical ToF cameras take four equallyspaced samples $y_{i}^{(n)}=g_{i}\left(\phi_{n} ; z_{i}\right)$ in each period, $\phi_{n}=n / 4 f$ for $n=0,1,2,3$. The rest of this paper will assume a 4-tuple $\mathbf{y}_{i}=\left(y_{i}^{(0)}, y_{i}^{(1)}, y_{i}^{(2)}, y_{i}^{(3)}\right)$ is obtained for each pixel $i$ at depth $z_{i}$ using modulation frequency $f$. The conventional processing gives pointwise estimates for sinusoid amplitude and wrapped distance:

$$
\begin{gathered}
\tilde{a}_{i}=\frac{1}{2} \sqrt{\left(y_{i}^{(0)}\right)^{2}+\left(y_{i}^{(1)}\right)^{2}+\left(y_{i}^{(2)}\right)^{2}+\left(y_{i}^{(3)}\right)^{2}}, \\
\tilde{z}_{i}=\frac{c}{4 \pi f} \tan ^{-1}\left(\frac{y_{i}^{(1)}-y_{i}^{(3)}}{y_{i}^{(0)}-y_{i}^{(2)}}\right) .
\end{gathered}
$$

\subsection{Statistical Distribution of Phase Measurements}

ToF cameras are affected by shot noise [23], which is due to the conversion of photons to electric charge in the sensor. This charge generation can be modeled as a time inhomogeneous Poisson process at each sensor pixel with rate $\lambda_{i}(t)=\eta r_{i}(t)$, where $\eta$ is the quantum efficiency of the sensor.

The correlation function is averaged over $N$ periods to counter the effects of the shot noise. For large $N$, according to the central limit theorem, the output is approximately normally distributed,

$$
y_{i}^{(n)} \mid z_{i} \sim \mathcal{N}\left(\eta g\left(\frac{n}{4 f} ; z_{i}\right), \frac{1}{2} \eta\left(a_{i}+b_{i}\right) / N\right) \text { for } n=0,1,2,3 .
$$

Each measurement of the cross correlation function is conditionally independently given $z_{i}$. The joint distribution of the measurements 4-tuple $\mathbf{y}_{i}$ given the true distance at pixel $i$ is $z_{i}$ is given by

$$
p\left(\mathbf{y}_{i} \mid z_{i}\right) \propto \prod_{n=0}^{3} \exp \left[-\frac{\left(\eta a \cos \left(\frac{1}{2} n \pi-4 \pi f c^{-1} z_{i}\right)-y_{i}^{(n)}\right)^{2}}{2 \sigma_{i}^{2}}\right],
$$

where $\sigma_{i}^{2}=\frac{1}{2} \eta\left(a_{i}+b_{i}\right) / N$. Then the likelihood on observations $\mathbf{y}_{i}$ given true distance $z_{i}$ takes the form

$$
p\left(\mathbf{y}_{i} \mid z_{i}\right)=\frac{C}{4 \pi^{2} \sigma_{i}^{4}} \exp \left\{-\frac{\eta a_{i} A_{i}}{\sigma_{i}^{2}} \cos \left(4 \pi f c^{-1}\left(z_{i}-\tilde{z}_{i}\right)\right)\right\},
$$

where $A_{i}=\sqrt{\left(y_{i}^{(0)}-y_{i}^{(2)}\right)^{2}+\left(y_{i}^{(1)}-y_{i}^{(3)}\right)^{2}}$ and $\tilde{z}_{i}$ is defined as in (2). Since $a_{i}$ is not available directly to form our likelihood, we instead use the value estimated as in (1), which is readily available from measurements taken in current ToF cameras.

\subsection{Phase Unwrapping}

Since ToF imaging uses a periodic modulation source, measurements beyond a certain range are ambiguous, and many different true depth values can produce the same measurement; specifically, $z_{i}=\tilde{z}_{i}+n c /(2 f)$ for any $n \in \mathbb{Z}$. The maximum unambiguous distance $D=c /(2 f)$ increases as modulation frequency decreases, but with noise, the error in estimating distance increases as well; this well-known fact can be derived from the earlier analysis.

Using two different modulation frequencies $f_{0}$ and $f_{1}$,

$$
z_{i}=\tilde{z}_{i 0}+n_{0} \frac{c}{2 f_{0}}=\tilde{z}_{i 1}+n_{1} \frac{c}{2 f_{1}} \quad \text { for } n_{0}, n_{1} \in \mathbb{Z}
$$

is the condition for ambiguity, where $\tilde{z}_{i j}$ is the measured distance at frequency $f_{j}$. From this we can see that the extended unambiguous distance is increased to $D=c /\left(2 \operatorname{gcd}\left(f_{0}, f_{1}\right)\right)$. In a noiseless setting, the true distance within the extended range can be computed by solving the above system. With noise, the unwrapping problem is nontrivial. Consider the joint likelihood of measuring both sets of four samples at each of two frequencies, $p\left(\mathbf{y}_{i 0}, \mathbf{y}_{i 1} \mid z_{i}\right)=$ $p\left(\mathbf{y}_{i 0} \mid z_{i}\right) p\left(\mathbf{y}_{i 1} \mid z_{i}\right)$. The value for $z_{i}$ that maximizes the likelihood function can be used as a pointwise estimate for the true distance of the scene point. However, this likelihood is highly nonconvex and finding the maximum likelihood estimate (MLE) essentially requires a global grid search within the extended unambiguous distance.

\section{UNWRAPPING AND DENOISING USING GAMP}

\subsection{Sparsity-Based Denoising}

Images of natural scenes have been shown to have sparse representations in certain bases, including discrete wavelet bases [24]. This sparsity applies also to depth maps and can be exploited to perform denoising. Wavelet coefficients $\mathbf{x}$ for a typical depth map tend to be approximately Laplacian in distribution, with density

$$
p\left(x_{k}\right)=\frac{1}{2 q} e^{-\left|x_{k} / q\right|}
$$

for some parameter $q$. In modeling wavelet coefficients of a noisy image, $q$ varies based on the scene structure and noise variance. One method for denoising an image is to perform soft thresholding on the wavelet coefficients [25]. This is equivalent to performing the maximum a posteriori probability estimate with a Laplacian prior on the coefficients assuming a Gaussian noise model.

\subsection{GAMP-Based Unwrapping and Denoising}

Fig. 2 shows a graphical model for our acquisition process. This is amenable to GAMP since wavelet coefficients $\mathbf{x}$ can be modeled as independent and the measurement process is separable on the spatial-domain quantities $\mathbf{z}=\boldsymbol{\Phi} \mathbf{x}$. Like approximate message passing [26] and earlier techniques, GAMP uses approximations for certain messages in loopy BP; unlike earlier techniques it allows the non-Gaussianity of noise and nonlinearities discussed in Section 2.

To incorporate our model into the GAMP framework, we have specified the prior distributions $p\left(x_{k}\right)$ and probabilistic measurement channels $p\left(\mathbf{y}_{0 i}, \mathbf{y}_{1 i} \mid z_{i}\right)$ as well as the linear transform matrix $\boldsymbol{\Phi}$. The distribution $p\left(x_{k}\right)$ given in (4) promotes sparsity of 


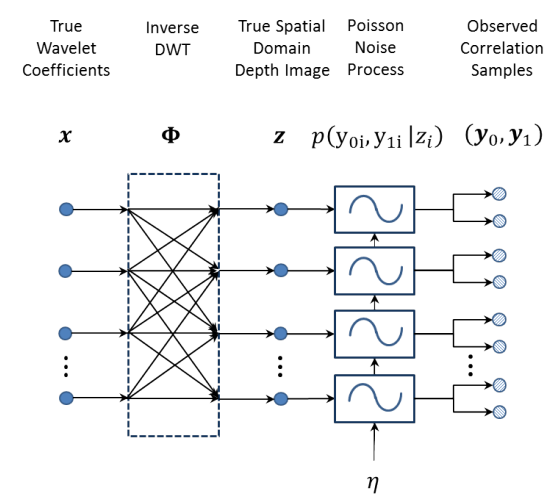

Fig. 2: Forward acquisition model for the ToF camera measurements at two modulation frequencies.

the wavelet coefficients of the estimate. The linear mixing matrix $\boldsymbol{\Phi}$ performs the inverse discrete wavelet transform on $\mathbf{x}$ to obtain the unwrapped image $\mathbf{z}=\boldsymbol{\Phi} \mathbf{x}$. The algorithm is summarized below, and one step is illustrated in Fig. 3.

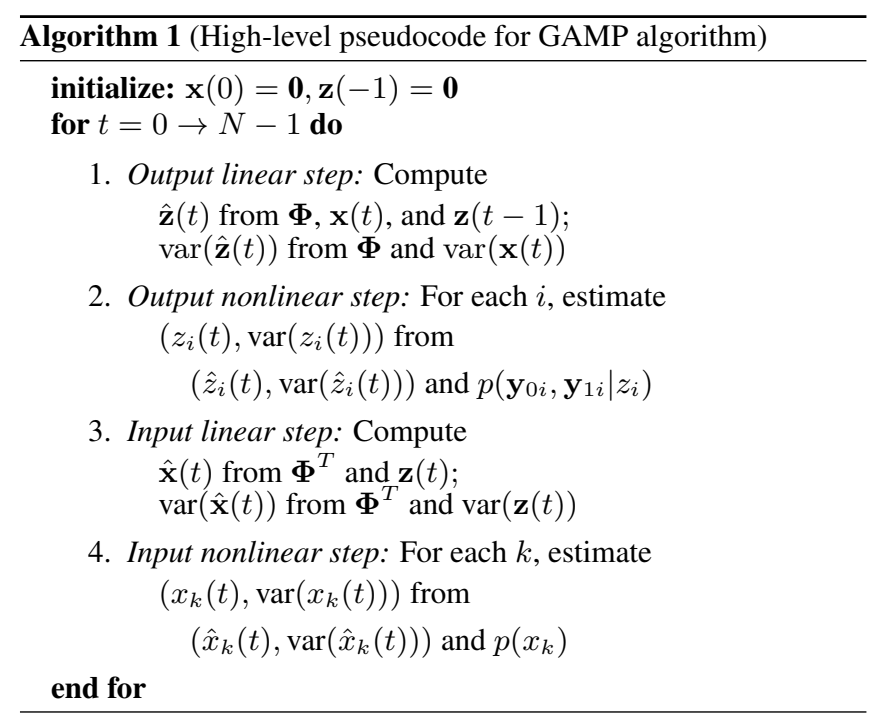

The output nonlinear step in Algorithm 1 estimates an expectation and variance for $z_{i}$ based on the sample vectors $\left(\mathbf{y}_{0 i}, \mathbf{y}_{1 i}\right)$, the likelihood function $p\left(\mathbf{y}_{0 i}, \mathbf{y}_{1 i} \mid z_{i}\right)$, and the previous computed values for the expectation and variance of $\hat{z}_{i}$ (see [19] for details). However, the exact integral required does not have a closed-form expression and is cumbersome to directly numerically integrate. Instead, an approximation can be made to the likelihood function to simplify computation. The exponential of a cosine from (3) can be closely approximated by the wrapped normal distribution [27]. For $j=0,1$ and each $i$, the approximated likelihood function is

$$
p\left(\mathbf{y}_{j i} \mid z_{i}\right) \propto \sum_{n=-\infty}^{\infty} \frac{1}{\sqrt{2 \pi} \sigma_{j i}} \exp \left\{-\frac{\left(z_{i}-\tilde{z}_{j i}+n D_{j}\right)^{2}}{2 \sigma_{j i}^{2}}\right\}
$$

where

$$
\sigma_{j i}^{2}=\frac{2 c^{2}}{\left(4 \pi f_{j}\right)^{2}} \ln \left(\frac{I_{0}\left(\eta a_{i} A_{j i} / \sigma_{i}^{2}\right)}{I_{1}\left(\eta a_{i} A_{j i} / \sigma_{i}^{2}\right)}\right)
$$

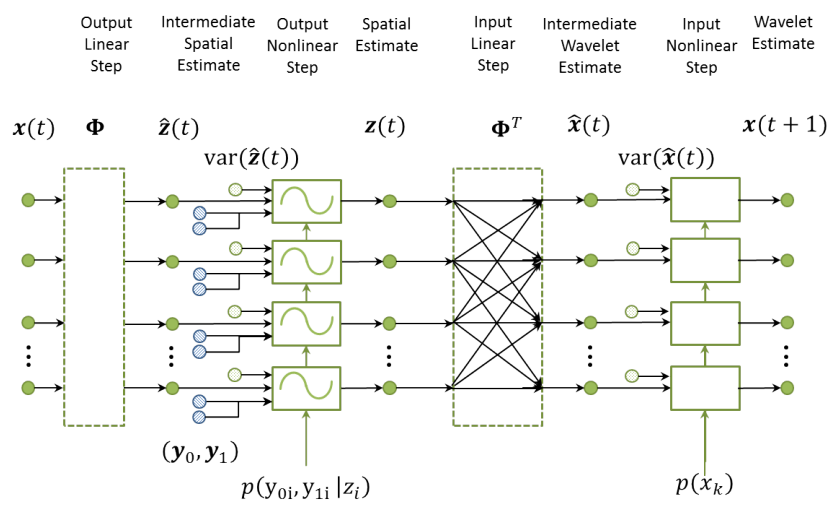

Fig. 3: One iteration of our GAMP algorithm for unwrapping and denoising. This diagram shows the updates of the estimates $\hat{\mathbf{z}}(t)$ and $\hat{\mathbf{x}}(t)$, represented by darker green nodes. The variances of the estimates, $\operatorname{var}(\hat{\mathbf{z}}(t))$ and $\operatorname{var}(\hat{\mathbf{x}}(t))$, represented by the light green nodes, are updated concurrently at each step in a similar computation.

and $I_{n}(k)$ is the modified Bessel function of the first kind. The likelihoods $p\left(\mathbf{y}_{0 i}, \mathbf{y}_{1 i} \mid z_{i}\right)$ and $p\left(\mathbf{y}_{0 i}, \mathbf{y}_{1 i} \mid z_{i}\right)$ can be further approximated without incurring much additional error within the extended unambiguous range by truncation to sums of $L$ and $M$ Gaussians respectively. The likelihood $p\left(\mathbf{y}_{0 i}, \mathbf{y}_{1 i} \mid z_{i}\right)$ then becomes a weighted sum of $L M$ Gaussians.

The use of the Gaussian mixture to approximate the true likelihood leads to a simple form for the expectation and variance computations in the output nonlinear step-much lower than numerical integration using the true likelihood. The linear mixing can be implemented using a 2D fast discrete wavelet transform. Furthermore, the structure of the algorithm allows the processing to be parallelized and optimized for computation in real time, implemented either in hardware or on a GPU, for example. Thus, the full integrated processing can be performed quickly and accurately.

\section{SIMULATIONS AND DISCUSSION}

Our integrated GAMP-based unwrapping and denoising method was compared against separate pointwise maximum likelihood estimation for unwrapping followed by wavelet thresholding using MatLab's default thresholds for denoising. The modulation frequencies chosen were $30 \mathrm{MHz}$ and $40 \mathrm{MHz}$, typical operating frequencies for ToF cameras, yielding a maximum unambiguous range of $c /(2 \cdot 10 \mathrm{MHz}) \approx 15 \mathrm{~m}$ from the gcd modulation frequency of 10 $\mathrm{MHz}$. The simulated scene was within the range of 0.5-12 m, causing wrapping for either modulation frequency taken separately.

The scene was the 450th frame taken from the right side camera of the Tsukuba stereo pair dataset under different illumination conditions $[28,29]$. The ground truth depth map was used to simulate $z$, the flashlight illumination represented active illumination and spatially varying reflectivity used to simulate amplitudes $a$, and the fluorescent light illumination simulated ambient background contributions $b$. Defining the SNR to be the ratio between the average amplitude of the sinusoid and the standard deviation of noise in the samples, SNR $=10 \log _{10}\left(2 a^{2} N /(a+b)\right) \mathrm{dB}$. We fix $a$ and $N=100$ and vary the SNR by varying $b$.

The 2D separable Daubechies length-4 discrete wavelet transform is used in both the baseline method and our method. Our GAMP algorithm was run for 20 iterations, and the MLE was found 


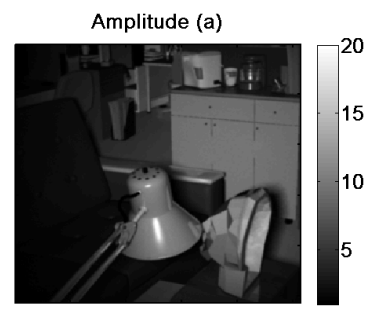

(a)

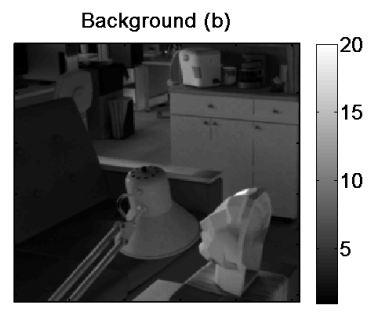

(b)

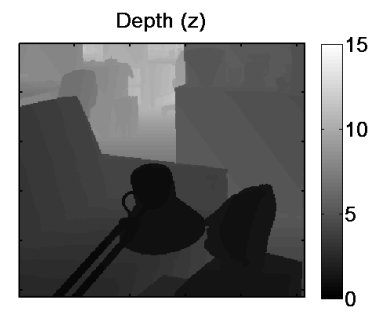

(c)

Fig. 4: Illumination conditions and ground truth for "Tsukuba 450" scene.

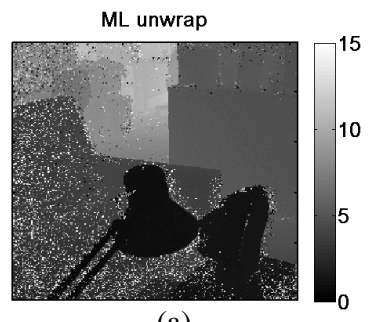

(a)

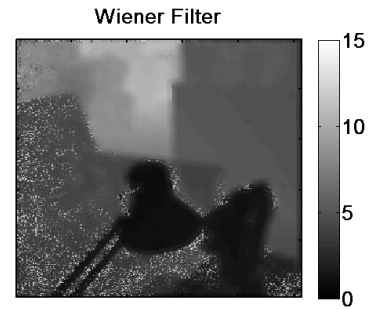

(e)

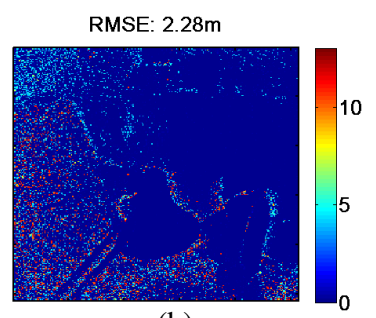

(b)

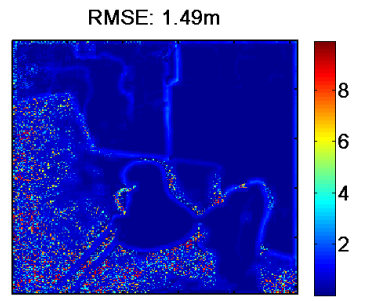

(f)

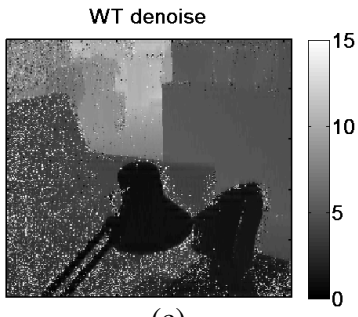

(c)

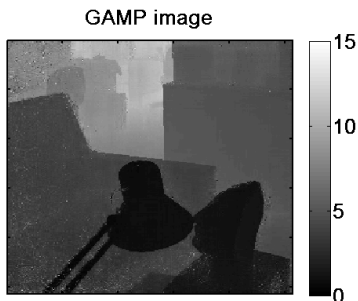

(g)

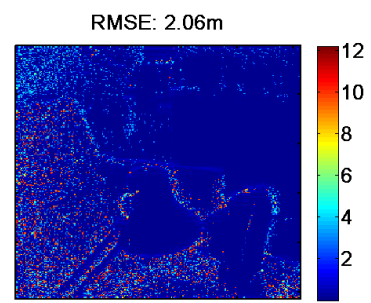

(d)

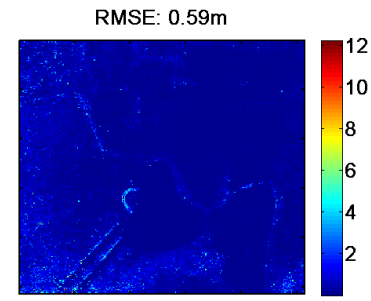

(h)

Fig. 5: Reconstructed depth maps and their root mean squared errors. The proposed GAMP method provides a $1.47 \mathrm{~m}$ improvement relative to the wavelet thresholded MLE, a $0.9 \mathrm{~m}$ improvement relative to the Wiener filtered MLE, and a 1.69m relative to the pointwise MLE. All images produced at $\mathrm{SNR}=10 \mathrm{~dB}$.

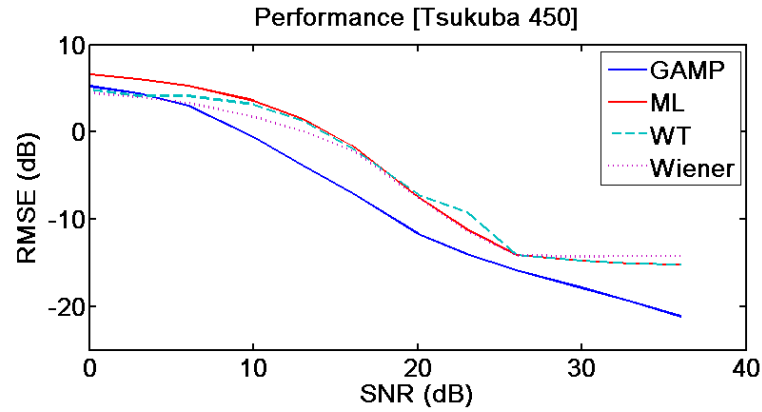

Fig. 6: MSE comparison for several methods across an SNR range.

using a discretized grid search. Wiener filtering was also performed using the estimated spectrum and noise variance of MLE depth map.

Fig. 4 shows the simulated scene along with the wrapped images produced by using (2) separately for each modulation frequency, with SNR $=10 \mathrm{~dB}$. Pointwise ML estimation is difficult (requiring a grid search) and does not perform well, as shown in Fig. 5(a,b). Post-processing the MLE by wavelet thresholding provides significant improvement, as shown in Fig. 5(c,d). Wiener filtering provides better MSE performance, as shown in Fig. 5(e,f). While the adaptive Wiener filter oversmooths in some blocks while leaving some of the nosier patches untouched, wavelet thresholding does not denoise enough of the image at this SNR. The proposed GAMP-based method does much better, both visually and in MSE, as shown in Fig. 5(g,h).

Fig. 6 provides a comparison over a range of low and moderate SNRs. At extremely low SNRs, all methods fail to produce a useful image; the proposed method allows successful operation at moderate SNRs, for which can increase robustness to ambient light, lower the illumination power requirement, or increase the frame rate. All simulation code is available at http://rleweb.mit.edu/stir/spud/.

\section{CONCLUSION}

We have proposed a new method for integrated processing of data in time-of-flight cameras to perform unwrapping and denoising jointly rather than separately. The result is greatly improved performance over post-processing of a noisy unwrapped image, particularly at low SNRs. Because the scene is within the extended unambiguous range, the success of the current method is due largely to the detailed acquisition modeling rather than the use of a signal prior. Future work could extend the integrated processing approach to beyond the unambiguous range, including to cases with a single modulation frequency, for certain classes of scenes. Automatic estimation of the parameters of a separable signal prior could be achieved by incorporating the Expectation-Maximization algorithm [30]. Greater structure in the signal prior could be incorporated using hybrid GAMP [31, 32]. 


\section{REFERENCES}

[1] A. Wehr and U. Lohr, "Airborne laser scanning—an introduction and overview," ISPRC J. Photogrammetry \& Remote Sensing, vol. 54, no. 2-3, pp. 68-82, Jul. 1999.

[2] B. Schwarz, "LIDAR: Mapping the world in 3D," Nature Photonics, vol. 4, no. 7, pp. 429-430, Jul. 2010.

[3] A. Medina, F. Gayá, and F. del Pozo, "Compact laser radar and three-dimensional camera," J. Opt. Soc. Amer. A., vol. 23, no. 4, pp. 800-805, Apr. 2006.

[4] S. Foix, G. Alenyà, and C. Torras, "Lock-in time-of-flight (ToF) cameras: A survey," IEEE Sensors J., vol. 11, no. 9, pp. 1917-1926, Sep. 2011.

[5] M. Hansard, S. Lee, O. Choi, and R. Horaud, Time-of-Flight Cameras: Principles, Methods and Applications, ser. SpringerBriefs in Computer Science. London: Springer, 2013.

[6] A. Kirmani, A. Colaço, F. N. C. Wong, and V. K. Goyal, "Exploiting sparsity in time-of-flight range acquisition using a single time-resolved sensor," Opt. Expr., vol. 19, no. 22, pp. 21 485-21 507, Oct. 2011.

[7] A. Colaço, A. Kirmani, G. A. Howland, J. C. Howell, and V. K. Goyal, "Compressive depth map acquisition using a single photon-counting detector: Parametric signal processing meets sparsity," in Proc. IEEE Conf. Comput. Vis. Pattern Recog., Providence, RI, Jun. 2012, pp. 96-102.

[8] L. Ying, "Phase unwrapping," in Wiley Encyclopedia of Biomedical Engineering, M. Akay, Ed. Wiley, 2006.

[9] D. C. Ghiglia and M. D. Pritt, Time-Dimensional Phase Unwrapping: Theory, Algorithms, and Software. New York: Wiley, 1998.

[10] M. D. Pritt and J. S. Shipman, "Least-squares two-dimensional phase unwrapping using FFT's," IEEE Trans. Signal Process., vol. 32, no. 3, pp. 706-708, May 1994.

[11] R. M. Goldstein, H. A. Zebker, and C. L. Werner, "Satellite radar interferometry: Two-dimensional phase unwrapping," Radio Sci., vol. 23, no. 4, pp. 713-720, Jan. 1988.

[12] N. H. Ching, D. Rosenfeld, and M. Braun, "Two-dimensional phase unwrapping using a minimum spanning tree algorithm," IEEE Trans. Image Process., vol. 1, no. 3, pp. 355-365, 1992.

[13] M. Costantini, "A novel phase unwrapping method based on network programming," IEEE Trans. Geosci. Remote Sensing, vol. 36, no. 3, pp. 813-821, May 1998.

[14] G. Valadão and J. Bioucas-Dias, "Phase imaging: Unwrapping and denoising with diversity and multi-resolution," in Proc. 2008 Int. Workshop on Local and Non-Local Approx. in Image Process., Lausanne, Switzerland, 2008.

[15] A. D. Payne, A. P. P. Jongenelen, A. A. Dorrington, M. J. Cree, and D. A. Carnegie, "Multiple frequency range imaging to remove measurement ambiguity," in Proc. 9th Conf. Optical 3-D Measurement Techniques, Vienna, Austria, Jul. 2009, pp. 139148.

[16] D. Droeschel, D. Holz, and S. Behnke, "Multi-frequency phase unwrapping for time-of-flight cameras," in IEEE/RSJ, 2010, pp. 1463-1469.

[17] A. Kirmani, A. Benedetti, and P. A. Chou.

[18] O. Choi and S. Lee, "Wide range stereo time-of-flight camera," in Proc. IEEE Int. Conf. Image Process., 2012.
[19] S. Rangan, "Generalized approximate message passing for estimation with random linear mixing," arXiv:1010.5141v1 [cs.IT]., Oct. 2010.

[20] _ - "Generalized approximate message passing for estimation with random linear mixing," in Proc. IEEE Int. Symp. Inform. Theory, Saint Petersburg, Russia, Jul.-Aug. 2011, pp. 2174-2178.

[21] P. Schniter and S. Rangan, "Compressive phase retrieval via generalized approximate message passing," in Proc. 50th Ann. Allerton Conf. on Commun., Control and Comp., Monticello, IL, Oct. 2012.

[22] S. B. Gokturk, H. Yalcin, and C. Bamji, "A time-of-flight depth sensor - system description, issues and solutions," in Proc. Conf. Comput. Vis. Pattern Recog. Workshop, 2004, p. 35.

[23] F. Mufti and R. Mahony, "Statistical analysis of measurement processes for time-of-flight cameras," in Proc. SPIE, vol. 7447, 2009, p. 74470I.

[24] A. Srivastava, A. B. Lee, E. P. Simoncelli, and S.-C. Zhu, "On advances in statistical modeling of natural images," J. Math. Imaging Vision, vol. 18, no. 1, pp. 17-33, Jan. 2003.

[25] D. L. Donoho, "De-noising by soft-thresholding," IEEE Trans. Inform. Theory, vol. 41, no. 3, pp. 613-627, May 1995.

[26] D. L. Donoho, A. Maleki, and A. Montanari, "Messagepassing algorithms for compressed sensing," Proc. Nat. Acad. Sci., vol. 106, no. 45, pp. 18914-18919, Nov. 2009.

[27] M. A. Stephens, "Random walk on a circle," Biometrika, vol. 50, no. 3-4, pp. 385-390, 1963.

[28] S. Martull, M. Peris, and K. Fukui, "Realistic cg stereo image dataset with ground truth disparity maps," ICPR workshop TrakMark2012, vol. 111, no. 430, pp. 117-118, 2012.

[29] M. Peris, S. Martull, A. Maki, Y. Ohkawa, and K. Fukui, “Towards a simulation driven stereo vision system," in Pattern Recognition (ICPR), 2012 21st International Conference on. IEEE, 2012, pp. 1038-1042.

[30] J. P. Vila and P. Schniter, "Expectation-maximization Gaussian-mixture approximate message passing," arXiv:1207.3107 [cs.IT], Jul. 2012.

[31] S. Rangan, A. K. Fletcher, V. K. Goyal, and P. Schniter, "Hybrid approximate message passing with applications to structured sparsity," arXiv:1111.2581 [cs.IT], Nov. 2011.

[32] — - "Hybrid generalized approximate message passing with applications to structured sparsity," in Proc. IEEE Int. Symp. Inform. Theory, Cambridge, MA, Jul. 2012, pp. 1241-1245. 